\title{
Influence of Ascorbic Acid and Calcium Chloride on Physical Parameters and Microbial Count of Firm Flesh Jackfruit Bulbs during Refrigerated Storage
}

\author{
Madhu M. Chulaki*, C.D. Pawar and Salma Mustafa Khan \\ Department of horticulture, DBSKKV, Dapoli, Maharashtra, India \\ *Corresponding author
}

A B S T R A C T

\begin{abstract}
Keywords
Ascorbic acid,

Calcium chloride,

KMS, Citric acid,

Chlorine water,

Physical parameters,

Microbial count,

Jackfruit bulbs.

Article Info

Accepted:

10 September 2017

Available Online:

10 October 2017

The present investigation on Influence of ascorbic acid and calcium chloride on physical parameters and microbial count of firm flesh jackfruit bulbs was carried out during 2014-15 at College of Agriculture, DBSKKV, Dapoli. A known quantity of jackfruit bulbs were treated with 12 different combinations of ascorbic acid $(0.0,0.5,1.0$ and $1.5 \%)$ and calcium chloride $(0.0,0.5$ and $1.0 \%)$ along with 100 ppm KMS, 0.5 percent citric acid and packed in thermocole tray, covered with permissible film and refrigerated. The treated jackfruit bulbs were analyzed for different physical parameters at two days interval. The treatment of 1.5 percent ascorbic acid, 0.5 percent calcium chloride with $100 \mathrm{ppm}$ KMS, 0.5 percent citric acid and surface sterilized with chlorine water $(30 \mathrm{ppm})$ resulted in extending the storage life of jackfruit bulbs upto 6 days with minimum PLW, colour change, moisture content, microbial count and spoilage in refrigerated condition $\left(6-7^{\circ} \mathrm{C}\right)$.
\end{abstract}

\section{Introduction}

Jackfruit (Artocarpus heterophyllus Lam.) one of the unexploited nutritious fruit indigenous to the rainforests of Western Ghats of India (Reddy et al., 2004). It was probably taken by Arab traders to the East African coast. Major producers are Bangladesh, India, Myanmar, Thailand, Vietnam, China, Philippines, Indonesia, Malaysia and Sri Lanka. India is the second biggest producer of the fruit in the world and is considered as the motherland of jackfruit. Jackfruit belongs to family Moraceae which encompasses about 1000 species in 67 genera, mostly tropical shrubs and trees, but also a few vines and herbs (Bailey, 1942; Merill, 1912).
The jackfruit, Artocarpus heterophyllus Lam. is also called as jack-fruit, jak and jaca. In Malaysia and Philippines called as nangka; in Thailand, khanun; in Combodia, Khnor; in Laos, mak mi or may mi and in Vietnam, mit. An edible portion of $100 \mathrm{~g}$ of (fresh weight) ripe jackfruit contains 72.0-94.0 g water, 1.2$1.9 \mathrm{~g}$ protein, 0.1-0.4 g fat, 16.0-25.4 g carbohydrate, $1.0-1.5 \mathrm{~g}$ fibre, $0.87-0.9 \mathrm{~g}$ total minerals, 20.0-37.0 mg calcium, 175-540 IU vitamin A, 88-410 $\mathrm{Kj}$ energy (Gunasena et al., 1996 and Azad, 2000).

Jackfruit is climacteric fruit having high degree of perishability. To minimize the postharvest losses and extend shelf life of 
jackfruit bulbs, minimal processing of jackfruit bulbs and provision of chemical treatment for bulbs is necessary. This will help to provide fresh bulbs in the hands of consumers. Minimal processing with chemicals like potassium metabisulphite (KMS), citric acid, calcium chloride and ascorbic acid helps to extend shelf life of fruits. Hence, the present study i.e., Influence of ascorbic acid and calcium chloride on physical parameters and microbial count of firm flesh jackfruit bulbs during refrigerated storage was undertaken to investigate the physical parameters and microbial count of minimally processed jackfruit bulbs.

\section{Materials and Methods}

For this study, ripe jackfruits of firm flesh type were collected and were kept for ripening at ambient temperature. All the chemicals used in this investigation were of analytical grade Thermocole tray and permissible film was used for packaging of jackfruit bulbs.

The ripe fruits were surface sterilized with $100 \mathrm{ppm}$ chlorinated water for 5 minutes and then cut opened. The jackfruit bulbs without removal of seeds were separated and whole, sound bulbs were used for experimentation. These bulbs were treated with $30 \mathrm{ppm}$ chlorinated water for 5 minutes. Again these bulbs were dipped in solution containing 100 ppm KMS, 0.5 percent citric acid, along with ascorbic acid (0.0, 0.5, 1.0 and 1.5\%) and calcium chloride $(0.0,0.5$ and 1.0 $\%)$ treatment combination, for 5 minutes.

These bulbs were blotted dry and 9 bulbs were packed per thermocole tray and covered with permissible plastic film. The packed bulbs were kept at refrigerated condition (6$7^{\circ} \mathrm{C}$ ) and observed for changes in physical and microbial count (bacterial and fungus) at two days interval (i.e., at every $3^{\text {rd }}$ day).
Physical parameter of jackfruit bulbs during storage

\section{Change in colour}

Change in colour of jackfruit bulbs was recorded by visual observations.

\section{Physiological loss in weight (PLW)}

For this study, the packaging materials were marked for studying physiological loss in weight from each treatment. The progressive loss in weight was noted at two days interval and percent loss in weight was calculated by using following formula.

$$
\% \mathrm{PLW}=\frac{\text { Initial weight }- \text { Final weight }}{\text { Initial weight }}
$$

\section{Moisture (\%)}

The per cent moisture in jackfruit bulbs was estimated by cabinet drying a known weight of sample. The sample was kept at $55-60{ }^{\circ} \mathrm{C}$ till constant weight was obtained.

The loss in weight per $100 \mathrm{~g}$ edible portion was calculated and reported as per cent moisture (A.O.A.C., 1975).

\section{Spoilage}

Each bulb from tray was thoroughly examined for any visible symptoms of spoilage during storage and spoilage percentage was calculated. The observations were recorded at two days interval.

\section{Shelf-life}

The shelf-life of jackfruit bulbs under study was computed on the basis of extent of spoilage of bulbs. The shelf life of bulbs was considered to be over when they showed 
spoilage upto an extent of 15 percent and above. Shelf life was recorded in days.

Microbial Count of jackfruit bulbs during storage

To estimate the bacterial and fungal growth in jackfruit bulbs stored at refrigeration condition, serial dilution and spread plate technique was followed using nutrient agar medium and potato dextrose agar medium, respectively. After 48 hours bacterial and fungal colonies were counted.

\section{Statistical analysis}

The data obtained during the present investigation were analyzed statistically in Factorial Completely Randomized Design (F.C.R.D) as per the method described by Panase and Sukhatme (1985).

\section{Results and Discussion}

\section{Change in colour}

It was revealed from the data (Table 1) that colour of jackfruit bulbs changes from yellow to yellowish brown with advancement of storage period. The bulbs of different treatments stored at refrigerated storage remain yellow up to 3 days of storage then the colour of treatments change to light yellow at $6^{\text {th }}$ day of storage. Further at $9^{\text {th }}$ day of storage all the treatments turn yellowish brown, which was an indication of severe spoilage.

\section{Physiological loss in weight (\%)}

The PLW of jackfruit bulbs (Table 2) increased throughout the storage period from 0.0 percent ( 0 days) to 8.02 percent ( 9 days), irrespective of ascorbic acid and calcium chloride treatments. With respect to ascorbic acid treatment, significant PLW was found to be decreased with increase in ascorbic acid concentration till $\mathrm{A}_{3}$ treatment and then increased at $\mathrm{A}_{4} \quad(3.31 \%$ and $8.22 \%)$ treatment at 6 and $9^{\text {th }}$ day, respectively. In case of calcium chloride treatment, PLW of jackfruit bulbs does not showed fixed trend with increase in calcium chloride concentration. Significant variation in PLW of jackfruit bulbs was shown by interaction of ascorbic acid and calcium chloride treatments. At $6^{\text {th }}$ day of storage lowest PLW was observed in $\mathrm{A}_{3} \mathrm{C}_{3}(2.99 \%)$ interaction and was at par with $\mathrm{A}_{1} \mathrm{C}_{3}(3.46 \%), \mathrm{A}_{2} \mathrm{C}_{3}(3.50$ $\%), \mathrm{A}_{3} \mathrm{C}_{2}(3.07 \%), \mathrm{A}_{4} \mathrm{C}_{1}(3.05 \%), \mathrm{A}_{4} \mathrm{C}_{2}$ $(3.58 \%)$ and $\mathrm{A}_{4} \mathrm{C}_{3}(3.31 \%)$ interactions. At $9^{\text {th }}$ day of storage lowest PLW was recorded in $\mathrm{A}_{3} \mathrm{C}_{2}(7.04 \%)$ interaction and was at par with $\mathrm{A}_{1} \mathrm{C}_{1}(7.72 \%)$ interaction.

\section{Moisture content}

The result presented in Table 3 revealed that, moisture of jackfruit bulbs decreased from 0 day $(72.74 \%)$ to $9^{\text {th }}$ day $(67.30 \%)$ of storage, irrespective of ascorbic acid and calcium chloride treatments. With respect to ascorbic acid treatment, the moisture content was found to be increased till $A_{2}$ treatments and then decreased till $\mathrm{A}_{4}$ treatment at 3,6 and $9^{\text {th }}$ day of storage. In case of calcium chloride treatments, moisture of jackfruit bulbs increased till $\mathrm{C}_{2}$ treatment and then decreased at $\mathrm{C}_{3}$ treatment during $6^{\text {th }}$ day of storage. At $9^{\text {th }}$ day of storage moisture decreased with increase in calcium chloride concentration, irrespective of ascorbic acid treatments. At $6^{\text {th }}$ day of storage, highest moisture was recorded in $\mathrm{A}_{2} \mathrm{C}_{2}(71.50 \%)$ and was at par with $\mathrm{A}_{2} \mathrm{C}_{1}$ $(69.95 \%), \mathrm{A}_{2} \mathrm{C}_{3}(70.80 \%)$ and $\mathrm{A}_{3} \mathrm{C}_{1}(69.95$ $\%)$ in case of interaction.

\section{Spoilage (\%)}

The data (Table 4) revealed that spoilage increased from 0.0 percent to ( 0 day) to 79.38 percent $\left(9^{\text {th }}\right.$ day), during refrigerated storage. In case of ascorbic acid treatment, spoilage decreased with increase in ascorbic acid 
concentration, except $A_{3}$ treatment at $6^{\text {th }}$ day of storage and at $9^{\text {th }}$ day of storage. In case of calcium chloride treatment, with increase in calcium chloride treatment spoilage decreased at $6^{\text {th }}$ day of storage. At $9^{\text {th }}$ day of storage spoilage decreased till $\mathrm{C}_{2}(76.08 \%)$ and then increased in $\mathrm{C}_{3}(80.72 \%)$ treatment, irrespective of ascorbic acid treatments. Significantly lowest spoilage was recorded in $\mathrm{A}_{4} \mathrm{C}_{2}(5.37 \%)$ interaction at $6^{\text {th }}$ day of storage and was superior over other interactions. At $9^{\text {th }}$ day of storage significantly lowest spoilage was observed in $\mathrm{A}_{2} \mathrm{C}_{1}(69.44 \%)$ interaction and was at par with $\mathrm{A}_{1} \mathrm{C}_{2}(75 \%)$, $\mathrm{A}_{2} \mathrm{C}_{3}(70.83 \%), \mathrm{A}_{4} \mathrm{C}_{1}(75 \%)$ and $\mathrm{A}_{4} \mathrm{C}_{2}$ (71.00\%) interactions.

\section{Shelf-life (days)}

The data presented in Table 5. Shelf life is calculated on the basis of spoilage percentage (Table 3). Significantly highest shelf-life (4.33 days) was recorded by $\mathrm{A}_{4}$ treatment and was significantly superior over other treatments in case of ascorbic acid treatment. In case of calcium chloride treatment, shelflife of jackfruit bulbs increased till $\mathrm{C}_{2}$ (3.75 days) treatment and decreased at $\mathrm{C}_{3}(3.25$ days) treatment. In case of interaction, it is evident from the data that maximum shelf life (6 days) was recorded in bulbs treated with combination of 1.5 percent of ascorbic acid and 0.5 percent of calcium chloride concentration i.e., $\mathrm{A}_{4} \mathrm{C}_{2}$ interaction and significantly superior over rest of the interactions. As far as effect of ascorbic acid and calcium chloride treatments at refrigerated condition on colour, PLW, spoilage and shelf life of firm flesh jackfruit bulbs is concerned, interaction $\mathrm{A}_{4} \mathrm{C}_{2}$ was found to be best.

\section{Bacterial and fungal count}

Bacterial (Table 6) and fungal count (Table 7) of fresh cut jackfruit bulbs increased throughout the storage period (upto $9^{\text {th }}$ day). At $9^{\text {th }}$ day of storage bacterial load decreased with increase in ascorbic acid treatment, except at $\mathrm{A}_{4}\left(15 \times 10^{-5}\right)$ treatment, irrespective of calcium chloride treatments.

Lowest bacterial count was recorded in $\mathrm{A}_{4}$ treatment at $9^{\text {th }}$ day of storage and results were non significant at 0,3 and $6^{\text {th }}$ day of storage. At $9^{\text {th }}$ day of storage bacterial load decreased till $\mathrm{C}_{2}\left(10.81 \times 10^{-5}\right)$ treatment and thereafter increased at $C_{3} \quad\left(15.62 \times 10^{-5}\right)$ treatment, irrespective of ascorbic acid treatments.

Lowest bacterial count was observed in $\mathrm{C}_{2}$ $\left(10.81 \times 10^{-5}\right)$ treatment.In case of interactions, $\mathrm{A}_{2} \mathrm{C}_{3}$ interaction recorded significantly lowest bacterial count at $9^{\text {th }}$ day of storage and results were non significant at other days of storage.

In case of fungal count results were non significant during entire storage period.

As far as effect of ascorbic acid and calcium chloride on microbial count is concerned, interaction $\mathrm{A}_{2} \mathrm{C}_{3}$ recorded lowest bacterial count followed by $\mathrm{A}_{3} \mathrm{C}_{2}$ and $\mathrm{A}_{4} \mathrm{C}_{2}$ at $9^{\text {th }}$ day of storage. In case of fungal count results were non significant.

\section{Change in colour}

The colour of jackfruit bulbs changes from yellow to yellowish brown with advancement of storage period was in accordance with the findings of Sexena et al., (2008) and Boodia et al., (2009) in jackfruit. Colour change may be indication of severe spoilage.

\section{Physiological loss in weight (\%)}

Increase in PLW with increase in storage period may be due to loss of moisture due to respiration and transpiration during storage. 
Table.1 Influence of ascorbic acid and calcium chloride on colour change of jackfruit bulbs during refrigerated storage

\begin{tabular}{|c|c|c|c|c|c|c|c|c|c|c|c|c|}
\hline \multicolumn{13}{|c|}{ Storage Days } \\
\hline \multirow{2}{*}{ Treatments } & \multicolumn{3}{|c|}{ 0 } & \multicolumn{3}{|c|}{3} & \multicolumn{3}{|c|}{6} & \multicolumn{3}{|c|}{9} \\
\hline & $\mathbf{C}_{1}$ & $\mathbf{C}_{2}$ & $\mathbf{C}_{3}$ & $\overline{C_{1}}$ & $\mathbf{C}_{2}$ & $\mathbf{C}_{3}$ & $\overline{C_{1}}$ & $\mathbf{C}_{2}$ & $\mathbf{C}_{3}$ & $\mathrm{C}_{1}$ & $\mathbf{C}_{2}$ & $\mathbf{C}_{3}$ \\
\hline $\mathbf{A}_{1}$ & Yellow & Yellow & Yellow & Yellow & Yellow & Yellow & $\begin{array}{c}\text { Light } \\
\text { Yellow }\end{array}$ & $\begin{array}{l}\text { Light } \\
\text { Yellow }\end{array}$ & $\begin{array}{l}\text { Light } \\
\text { Yellow }\end{array}$ & $\begin{array}{c}\text { Yellowish } \\
\text { brown }\end{array}$ & $\begin{array}{c}\text { Yellowish } \\
\text { brown }\end{array}$ & $\begin{array}{c}\text { Yellowish } \\
\text { brown }\end{array}$ \\
\hline $\mathbf{A}_{2}$ & Yellow & Yellow & Yellow & Yellow & Yellow & Yellow & $\begin{array}{l}\text { Light } \\
\text { Yellow }\end{array}$ & $\begin{array}{l}\text { Light } \\
\text { Yellow }\end{array}$ & $\begin{array}{l}\text { Light } \\
\text { Yellow }\end{array}$ & $\begin{array}{c}\text { Yellowish } \\
\text { brown }\end{array}$ & $\begin{array}{c}\text { Yellowish } \\
\text { brown }\end{array}$ & $\begin{array}{c}\text { Yellowish } \\
\text { brown }\end{array}$ \\
\hline $\mathbf{A}_{3}$ & Yellow & Yellow & Yellow & Yellow & Yellow & Yellow & $\begin{array}{c}\text { Light } \\
\text { Yellow }\end{array}$ & $\begin{array}{l}\text { Light } \\
\text { Yellow }\end{array}$ & $\begin{array}{c}\text { Light } \\
\text { Yellow }\end{array}$ & $\begin{array}{c}\text { Yellowish } \\
\text { brown }\end{array}$ & $\begin{array}{c}\text { Yellowish } \\
\text { brown }\end{array}$ & $\begin{array}{c}\text { Yellowish } \\
\text { brown }\end{array}$ \\
\hline $\mathbf{A}_{4}$ & Yellow & Yellow & Yellow & Yellow & Yellow & Yellow & $\begin{array}{l}\text { Light } \\
\text { Yellow }\end{array}$ & $\begin{array}{l}\text { Light } \\
\text { Yellow }\end{array}$ & $\begin{array}{l}\text { Light } \\
\text { Yellow }\end{array}$ & $\begin{array}{c}\text { Yellowish } \\
\text { brown }\end{array}$ & $\begin{array}{c}\text { Yellowish } \\
\text { brown }\end{array}$ & $\begin{array}{c}\text { Yellowish } \\
\text { brown }\end{array}$ \\
\hline
\end{tabular}

A1: $0.0 \%$ Ascorbic Acid A2: $0.5 \%$ Ascorbic Acid A3: 1.0\% Ascorbic Acid A4: 1.5\% Ascorbic Acid
C1: $0.0 \%$ Calcium Chloride
C2: $0.5 \%$ Calcium Chloride
C3:1.0\% Calcium Chloride

Table.2 Influence of ascorbic acid and calcium chloride on PLW (\%) of jackfruit bulbs during refrigerated storage

\begin{tabular}{|c|c|c|c|c|c|c|c|c|c|c|c|c|c|c|c|c|}
\hline \multicolumn{17}{|c|}{ Storage Days } \\
\hline \multirow{2}{*}{ Treatments } & \multicolumn{4}{|c|}{$\mathbf{0}$} & \multicolumn{4}{|c|}{3} & \multicolumn{4}{|c|}{6} & \multicolumn{4}{|c|}{9} \\
\hline & $\mathrm{C}_{1}$ & $\mathrm{C}_{2}$ & $\mathrm{C}_{3}$ & Mean & $\mathrm{C}_{1}$ & $\mathrm{C}_{2}$ & $\mathbf{C}_{3}$ & Mean & $\mathbf{C}_{1}$ & $\mathbf{C}_{2}$ & $\mathrm{C}_{3}$ & Mean & $\mathrm{C}_{1}$ & $\mathrm{C}_{2}$ & $\mathrm{C}_{3}$ & Mean \\
\hline $\mathbf{A}_{1}$ & 0.00 & 0.00 & 0.00 & 0.00 & 1.60 & 2.75 & 1.64 & 1.99 & 5.25 & 3.90 & 3.46 & 4.20 & 7.72 & 7.88 & 8.58 & 8.06 \\
\hline $\mathbf{A}_{2}$ & 0.00 & 0.00 & 0.00 & 0.00 & 1.52 & 2.49 & 1.68 & 1.90 & 4.56 & 3.77 & 3.50 & 3.94 & 7.36 & 8.74 & 8.77 & 8.29 \\
\hline $\mathbf{A}_{3}$ & 0.00 & 0.00 & 0.00 & 0.00 & 2.10 & 1.48 & 1.41 & 1.66 & 3.68 & 3.07 & 2.99 & 3.24 & 7.84 & 7.04 & 7.69 & 7.52 \\
\hline $\mathbf{A}_{4}$ & 0.00 & 0.00 & 0.00 & 0.00 & 1.50 & 1.36 & 1.75 & 1.53 & 3.05 & 3.58 & 3.31 & 3.31 & 7.89 & 8.16 & 8.61 & 8.22 \\
\hline \multirow[t]{2}{*}{ Mean } & 0.00 & 0.00 & 0.00 & 0.00 & 1.68 & 2.02 & 1.62 & 1.77 & 4.13 & 3.58 & 3.31 & 3.67 & 7.70 & 7.95 & 8.41 & 8.02 \\
\hline & \multicolumn{2}{|c|}{ SEm \pm} & \multicolumn{2}{|c|}{ C.D. at $1 \%$} & \multicolumn{2}{|c|}{ SEm \pm} & \multicolumn{2}{|c|}{ C.D. at 1\% } & \multicolumn{2}{|c|}{ SEm \pm} & \multicolumn{2}{|c|}{ C.D. at 1\% } & \multicolumn{2}{|c|}{ SEm \pm} & \multicolumn{2}{|c|}{ C.D. at 1\% } \\
\hline Ascorbic Acid & \multicolumn{2}{|c|}{-} & \multicolumn{2}{|c|}{-} & \multicolumn{2}{|c|}{0.090} & \multicolumn{2}{|c|}{0.262} & \multicolumn{2}{|c|}{0.135} & \multicolumn{2}{|c|}{0.395} & \multicolumn{2}{|c|}{0.135} & \multicolumn{2}{|c|}{0.395} \\
\hline $\begin{array}{l}\text { Calcium } \\
\text { Chloride }\end{array}$ & \multicolumn{2}{|c|}{-} & \multicolumn{2}{|c|}{-} & \multicolumn{2}{|c|}{0.077} & \multicolumn{2}{|c|}{0.227} & \multicolumn{2}{|c|}{0.117} & \multicolumn{2}{|c|}{0.342} & \multicolumn{2}{|c|}{0.117} & \multicolumn{2}{|c|}{0.342} \\
\hline $\mathbf{A x C}$ & \multicolumn{2}{|c|}{-} & \multicolumn{2}{|c|}{ - } & \multicolumn{2}{|c|}{0.155} & \multicolumn{2}{|c|}{0.455} & & & & 684 & & & & 684 \\
\hline
\end{tabular}

A1: 0.0\% Ascorbic Acid A2: $0.5 \%$ Ascorbic Acid A3: $1.0 \%$ Ascorbic Acid A4: 1.5\% Ascorbic Acid

C1: $0.0 \%$ Calcium Chloride $\quad$ C2: $0.5 \%$ Calcium Chloride $\quad$ C3:1.0 \% Calcium Chloride 
Table.3 Influence of ascorbic acid and calcium chloride on moisture (\%) of jackfruit bulbs during refrigerated storage

\begin{tabular}{|c|c|c|c|c|c|c|c|c|c|c|c|c|c|c|c|c|}
\hline \multicolumn{17}{|c|}{ Storage Days } \\
\hline \multirow[t]{2}{*}{ Treatments } & \multicolumn{4}{|c|}{$\mathbf{0}$} & \multicolumn{4}{|c|}{3} & \multicolumn{4}{|c|}{6} & \multicolumn{4}{|c|}{9} \\
\hline & $\mathrm{C}_{1}$ & $\mathrm{C}_{2}$ & $\mathrm{C}_{3}$ & Mean & $\overline{C_{1}}$ & $\mathrm{C}_{2}$ & $\mathbf{C}_{3}$ & Mean & $\overline{C_{1}}$ & $\mathrm{C}_{2}$ & $\mathbf{C}_{3}$ & Mean & $\overline{C_{1}}$ & $\mathbf{C}_{2}$ & $\mathbf{C}_{3}$ & Mean \\
\hline$\overline{\mathbf{A}_{1}}$ & 73.40 & 71.05 & 71.05 & 71.83 & 71.90 & 70.95 & 70.05 & 70.96 & 69.30 & 69.55 & 69.45 & 69.43 & 67.65 & 67.51 & 67.55 & 67.57 \\
\hline $\mathbf{A}_{2}$ & 71.20 & 74.25 & 76.55 & 74.00 & 70.50 & 71.50 & 71.45 & 71.15 & 69.95 & 71.50 & 70.80 & 70.75 & 69.85 & 67.80 & 68.50 & 68.71 \\
\hline $\mathbf{A}_{3}$ & 73.75 & 72.05 & 70.90 & 72.23 & 71.80 & 70.95 & 70.60 & 71.11 & 69.95 & 71.05 & 66.10 & 69.03 & 68.05 & 68.20 & 64.60 & 66.95 \\
\hline $\mathbf{A}_{4}$ & 71.20 & 74.25 & 73.25 & 72.9 & 70.65 & 72.65 & 69.95 & 71.08 & 68.75 & 69.65 & 67.90 & 68.76 & 67.70 & 65.90 & 64.35 & 65.98 \\
\hline \multirow[t]{2}{*}{ Mean } & 72.38 & 72.90 & 72.93 & 72.74 & 71.21 & 71.51 & 70.51 & 71.07 & 69.48 & 70.43 & 68.56 & 69.49 & 68.31 & 67.35 & 66.25 & 67.30 \\
\hline & \multicolumn{2}{|c|}{ SEm \pm} & \multicolumn{2}{|c|}{ C.D. at 1\% } & \multicolumn{2}{|c|}{ SEm \pm} & \multicolumn{2}{|c|}{ C.D. at 1\% } & \multicolumn{2}{|c|}{ SEm \pm} & \multicolumn{2}{|c|}{ C.D. at $1 \%$} & \multicolumn{2}{|c|}{ SEm \pm} & \multicolumn{2}{|c|}{ C.D. at $1 \%$} \\
\hline Ascorbic Acid & \multicolumn{2}{|c|}{0.372} & \multicolumn{2}{|c|}{1.087} & \multicolumn{2}{|c|}{0.372} & \multicolumn{2}{|c|}{$\mathrm{NS}$} & \multicolumn{2}{|c|}{0.374} & \multicolumn{2}{|c|}{1.087} & \multicolumn{2}{|c|}{0.337} & \multicolumn{2}{|c|}{0.985} \\
\hline $\begin{array}{l}\text { Calcium } \\
\text { Chloride }\end{array}$ & \multicolumn{2}{|c|}{0.356} & \multicolumn{2}{|c|}{ NS } & \multicolumn{2}{|c|}{0.322} & \multicolumn{2}{|c|}{ NS } & \multicolumn{2}{|c|}{0.323} & \multicolumn{2}{|c|}{0.945} & \multicolumn{2}{|c|}{0.292} & \multicolumn{2}{|c|}{0.853} \\
\hline$\overline{A x C}$ & \multicolumn{2}{|c|}{0.713} & \multicolumn{2}{|c|}{2.082} & \multicolumn{2}{|c|}{0.645} & \multicolumn{2}{|c|}{ NS } & & & & & & & & 07 \\
\hline
\end{tabular}

A1: $0.0 \%$ Ascorbic Acid A2: $0.5 \%$ Ascorbic Acid A3: 1.0\% Ascorbic Acid A4: 1.5\% Ascorbic Acid
C1: $0.0 \%$ Calcium Chloride
C2: $0.5 \%$ Calcium Chloride
C3:1.0 \% Calcium Chloride

Table.4 Influence of ascorbic acid and calcium chloride on spoilage (\%) of jackfruit bulbs during refrigerated storage

\begin{tabular}{|c|c|c|c|c|c|c|c|c|c|c|c|c|c|c|c|c|}
\hline \multicolumn{17}{|c|}{ Storage Days } \\
\hline \multirow[t]{2}{*}{ Treatments } & \multicolumn{4}{|c|}{$\mathbf{0}$} & \multicolumn{4}{|c|}{3} & \multicolumn{4}{|c|}{6} & \multicolumn{4}{|c|}{9} \\
\hline & $\mathbf{C}_{1}$ & $\mathbf{C}_{2}$ & $\mathbf{C}_{3}$ & Mean & $\mathrm{C}_{1}$ & $\mathbf{C}_{2}$ & $\mathbf{C}_{3}$ & Mean & $\mathrm{C}_{1}$ & $\mathrm{C}_{2}$ & $\mathbf{C}_{3}$ & Mean & $\mathrm{C}_{1}$ & $\mathrm{C}_{2}$ & $\mathbf{C}_{3}$ & Mean \\
\hline$\overline{A_{1}}$ & 0.00 & 0.00 & 0.00 & 0.00 & 0.00 & 0.00 & 0.00 & 0.00 & 51.38 & 40.97 & 33.33 & 41.89 & 100.0 & 75.00 & 83.33 & 86.11 \\
\hline $\mathbf{A}_{2}$ & 0.00 & 0.00 & 0.00 & 0.00 & 0.00 & 0.00 & 0.00 & 0.00 & 28.47 & 34.72 & 22.22 & 28.47 & 69.44 & 81.94 & 70.83 & 74.07 \\
\hline $\mathbf{A}_{3}$ & 0.00 & 0.00 & 0.00 & 0.00 & 0.00 & 0.00 & 0.00 & 0.00 & 34.02 & 51.38 & 29.16 & 38.19 & 81.25 & 76.38 & 81.25 & 79.62 \\
\hline $\mathbf{A}_{4}$ & 0.00 & 0.00 & 0.00 & 0.00 & 0.00 & 0.00 & 0.00 & 0.00 & 51.38 & 5.37 & 17.36 & 24.70 & 75.00 & 71.00 & 87.50 & 77.83 \\
\hline \multirow[t]{2}{*}{ Mean } & 0.00 & 0.00 & 0.00 & 0.00 & 0.00 & 0.00 & 0.00 & 0.00 & 41.31 & 33.11 & 25.51 & 33.31 & 81.42 & 76.08 & 80.72 & 79.38 \\
\hline & \multicolumn{2}{|c|}{ SEm \pm} & \multicolumn{2}{|c|}{ C.D. at $1 \%$} & \multicolumn{2}{|c|}{ SEm \pm} & \multicolumn{2}{|c|}{ C.D. at $1 \%$} & \multicolumn{2}{|c|}{ SEm \pm} & \multicolumn{2}{|c|}{ C.D. at $1 \%$} & \multicolumn{2}{|c|}{ SEm \pm} & \multicolumn{2}{|c|}{ C.D. at 1\% } \\
\hline Ascorbic Acid & \multicolumn{2}{|c|}{-} & \multicolumn{2}{|c|}{-} & \multicolumn{2}{|c|}{-} & \multicolumn{2}{|c|}{-} & \multicolumn{2}{|c|}{1.524} & \multicolumn{2}{|c|}{4.448} & \multicolumn{2}{|c|}{1.395} & \multicolumn{2}{|c|}{3.954} \\
\hline $\begin{array}{l}\text { Calcium } \\
\text { Chloride }\end{array}$ & \multicolumn{2}{|c|}{-} & \multicolumn{2}{|r|}{ - } & \multicolumn{2}{|c|}{-} & \multicolumn{2}{|c|}{ - } & \multicolumn{2}{|c|}{1.319} & \multicolumn{2}{|c|}{3.852} & \multicolumn{2}{|c|}{1.208} & \multicolumn{2}{|c|}{3.424} \\
\hline $\mathrm{AxC}$ & \multicolumn{2}{|c|}{ - } & \multicolumn{2}{|c|}{-} & \multicolumn{2}{|c|}{-} & \multicolumn{2}{|c|}{ - } & & & & & & & & 49 \\
\hline
\end{tabular}

A1: $0.0 \%$ Ascorbic Acid A2: $0.5 \%$ Ascorbic Acid A3: $1.0 \%$ Ascorbic Acid A4: $1.5 \%$ Ascorbic Acid

C1: $0.0 \%$ Calcium Chloride $\quad$ C2: $0.5 \%$ Calcium Chloride $\quad$ C3:1.0\% Calcium Chloride 
Table.5 Influence of ascorbic acid and calcium chloride on Shelf life (Days) of jackfruit bulbs during refrigerated storage

\begin{tabular}{|c|c|c|c|c|}
\hline \multirow[t]{2}{*}{ Treatments } & \multicolumn{4}{|c|}{ Shelf life (Days) } \\
\hline & $\mathbf{C}_{1}$ & $\mathbf{C}_{2}$ & $\mathbf{C}_{3}$ & Mean \\
\hline $\mathbf{A}_{1}$ & 3 & 3 & 3 & 3 \\
\hline $\mathbf{A}_{2}$ & 3 & 3 & 3 & 3 \\
\hline $\mathbf{A}$ & 3 & 3 & 3 & 3 \\
\hline $\mathbf{A}_{4}$ & 3 & 6 & 4 & 4.33 \\
\hline \multirow[t]{2}{*}{ Mean } & 3 & 3.75 & 3.25 & 3.33 \\
\hline & \multicolumn{2}{|c|}{ SEm \pm} & \multicolumn{2}{|c|}{ C.D. at $1 \%$} \\
\hline Ascorbic Acid & \multicolumn{2}{|c|}{0.166} & \multicolumn{2}{|c|}{0.486} \\
\hline Calcium Chloride & \multicolumn{2}{|c|}{0.144} & \multicolumn{2}{|c|}{0.421} \\
\hline $\mathrm{AxC}$ & \multicolumn{2}{|c|}{0.288} & \multicolumn{2}{|c|}{0.842} \\
\hline
\end{tabular}

A1: $0.0 \%$ Ascorbic Acid A2: $0.5 \%$ Ascorbic Acid A3: $1.0 \%$ Ascorbic Acid A4: $1.5 \%$ Ascorbic Acid

C1: $0.0 \%$ Calcium Chloride $\quad$ C2: $0.5 \%$ Calcium Chloride $\quad$ C3:1.0\% Calcium Chloride

Table.6 Influence of ascorbic acid and calcium chloride on Bacterial count $\left(10^{-5}\right)$ of jackfruit bulbs during refrigerated storage

\begin{tabular}{|c|c|c|c|c|c|c|c|c|c|c|c|c|c|c|c|c|}
\hline \multicolumn{17}{|c|}{ Storage Days } \\
\hline \multirow[t]{2}{*}{ Treatments } & \multicolumn{4}{|c|}{$\mathbf{0}$} & \multicolumn{4}{|c|}{3} & \multicolumn{4}{|c|}{6} & \multicolumn{4}{|c|}{9} \\
\hline & $\mathrm{C}_{1}$ & $\mathrm{C}_{2}$ & $\overline{C_{3}}$ & Mean & $\mathrm{C}_{1}$ & $\overline{C_{2}}$ & $\mathrm{C}_{3}$ & Mean & $\overline{C_{1}}$ & $\mathrm{C}_{2}$ & $\mathrm{C}_{3}$ & Mean & $\mathrm{C}_{1}$ & $\overline{C_{2}}$ & $\overline{C_{3}}$ & Mean \\
\hline $\mathbf{A}_{1}$ & 0.00 & 0.00 & 0.00 & 0.00 & 1.50 & 0.00 & 0.50 & 0.66 & 2.00 & 0.50 & 2.50 & 1.66 & 21.00 & 6.25 & 25.50 & 17.58 \\
\hline $\mathbf{A}_{2}$ & 0.00 & 0.50 & 0.00 & 0.16 & 1.00 & 0.50 & 0.00 & 0.50 & 1.50 & 1.00 & 1.00 & 1.16 & 16.50 & 15.00 & 7.00 & 12.83 \\
\hline $\mathbf{A}_{3}$ & 0.00 & 0.00 & 0.00 & 0.00 & 0.50 & 0.50 & 0.50 & 0.50 & 1.75 & 1.00 & 1.00 & 1.25 & 17.00 & 10.50 & 17.50 & 15.00 \\
\hline $\mathbf{A}_{4}$ & 0.00 & 0.00 & 0.00 & 0.00 & 0.50 & 0.00 & 0.50 & 0.33 & 1.50 & 0.50 & 2.50 & 1.50 & 14.00 & 11.50 & 12.50 & 12.66 \\
\hline \multirow[t]{2}{*}{ Mean } & 0.00 & 0.12 & 0.00 & 0.04 & 0.87 & 0.25 & 0.37 & 0.50 & 1.68 & 0.75 & 1.75 & 1.39 & 17.12 & 10.81 & 15.62 & 14.52 \\
\hline & \multicolumn{2}{|c|}{ SEm \pm} & \multicolumn{2}{|c|}{ C.D. at 1\% } & \multicolumn{2}{|c|}{ SEm \pm} & \multicolumn{2}{|c|}{ C.D. at 1\% } & \multicolumn{2}{|c|}{ SEm \pm} & \multicolumn{2}{|c|}{ C.D. at 1\% } & \multicolumn{2}{|c|}{ SEm \pm} & \multicolumn{2}{|c|}{ C.D. at 1\% } \\
\hline Ascorbic Acid & \multicolumn{2}{|c|}{0.048} & \multicolumn{2}{|c|}{ NS } & \multicolumn{2}{|c|}{0.166} & \multicolumn{2}{|c|}{ NS } & \multicolumn{2}{|c|}{0.312} & \multicolumn{2}{|c|}{ NS } & \multicolumn{2}{|c|}{0.277} & \multicolumn{2}{|c|}{0.809} \\
\hline $\begin{array}{l}\text { Calcium } \\
\text { Chloride }\end{array}$ & \multicolumn{2}{|c|}{0.041} & \multicolumn{2}{|c|}{ NS } & \multicolumn{2}{|c|}{0.144} & \multicolumn{2}{|c|}{ NS } & \multicolumn{2}{|c|}{0.270} & \multicolumn{2}{|c|}{ NS } & \multicolumn{2}{|c|}{0.240} & \multicolumn{2}{|c|}{0.701} \\
\hline $\mathrm{AxC}$ & \multicolumn{2}{|c|}{0.083} & \multicolumn{2}{|c|}{ NS } & \multicolumn{2}{|c|}{0.288} & \multicolumn{2}{|c|}{$\mathrm{NS}$} & 0.5 & & & $S$ & 0. & & & 02 \\
\hline
\end{tabular}

A1: $0.0 \%$ Ascorbic Acid A2: $0.5 \%$ Ascorbic Acid A3: $1.0 \%$ Ascorbic Acid A4: $1.5 \%$ Ascorbic Acid

$\begin{array}{lll}\mathrm{C} 1: 0.0 \% \text { Calcium Chloride } \quad \mathrm{C} 2: 0.5 \% \text { Calcium Chloride } & \mathrm{C} 3: 1.0 \% \text { Calcium Chloride }\end{array}$ 
Table.7 Influence of ascorbic acid and calcium chloride on fungal count $\left(10^{-4}\right)$ of jackfruit bulbs during refrigerated storage.

\begin{tabular}{|c|c|c|c|c|c|c|c|c|c|c|c|c|c|c|c|c|}
\hline \multicolumn{17}{|c|}{ Storage Days } \\
\hline Treatments & \multicolumn{4}{|c|}{$\mathbf{0}$} & \multicolumn{4}{|c|}{3} & \multicolumn{4}{|c|}{6} & \multicolumn{4}{|c|}{9} \\
\hline $\mathbf{A}_{1}$ & 0.00 & 0.00 & 0.00 & 0.00 & 0.00 & 0.00 & 0.00 & 0.00 & 1.00 & 0.75 & 1.00 & 0.91 & 3.00 & 1.75 & 1.00 & 1.91 \\
\hline$\overline{A_{3}}$ & 0.00 & 0.00 & 0.00 & 0.00 & 0.00 & 0.00 & 0.00 & 0.00 & 0.50 & 0.00 & 0.50 & 0.33 & 1.00 & 3.00 & 1.00 & 1.66 \\
\hline $\mathbf{A}_{4}$ & 0.00 & 0.00 & 0.00 & 0.00 & 0.00 & 0.00 & 0.00 & 0.00 & 0.00 & 0.00 & 0.00 & 0.00 & 1.25 & 1.00 & 0.50 & 0.91 \\
\hline Mean & \multicolumn{2}{|c|}{ SEm \pm} & \multicolumn{2}{|c|}{ C.D. at $1 \%$} & \multicolumn{2}{|c|}{ SEm \pm} & \multicolumn{2}{|c|}{ C.D. at $1 \%$} & \multicolumn{2}{|c|}{ SEm \pm} & \multicolumn{2}{|c|}{ C.D. at $1 \%$} & \multicolumn{2}{|c|}{ SEm \pm} & \multicolumn{2}{|c|}{ C.D. at $1 \%$} \\
\hline Ascorbic Acid & \multicolumn{2}{|c|}{-} & & - & \multicolumn{2}{|c|}{-} & \multicolumn{2}{|c|}{-} & \multicolumn{2}{|c|}{0.146} & \multicolumn{2}{|c|}{0.427} & \multicolumn{2}{|c|}{0.335} & \multicolumn{2}{|c|}{ NS } \\
\hline $\begin{array}{l}\text { Calcium } \\
\text { Chloride }\end{array}$ & \multicolumn{2}{|c|}{-} & \multicolumn{2}{|r|}{-} & \multicolumn{2}{|c|}{-} & \multicolumn{2}{|c|}{-} & \multicolumn{2}{|c|}{0.126} & \multicolumn{2}{|c|}{ NS } & \multicolumn{2}{|c|}{0.290} & \multicolumn{2}{|c|}{ NS } \\
\hline $\mathrm{AxC}$ & \multicolumn{2}{|c|}{-} & & - & \multicolumn{2}{|c|}{-} & \multicolumn{2}{|r|}{ - } & \multicolumn{2}{|c|}{0.253} & \multicolumn{2}{|c|}{ NS } & 0.5 & & & NS \\
\hline
\end{tabular}


Observation analogous to present finding was reported by Phutankar (2013) in jackfruit bulbs. Singh et al., (2014) reported that PLW of guava decreased significantly with the increase in concentration of ascorbic acid from $25 \mathrm{ppm}$ to $100 \mathrm{ppm}$. Lowest PLW at higher concentration $\left(\mathrm{C}_{3}\right)$ of calcium chloride at $3^{\text {rd }}$ and $6^{\text {th }}$ day of storage may be due to calcium application, which has been reported to be effective in terms of membrane integrity maintenance with lower losses of phospholipids and proteins and reduced ion leakage which could be responsible for the lower weight loss. Mahajan et al., (2011) used calcium chloride concentration of 1 per cent, 2 percent and 3 percent in guava fruits and found similar results. At $6^{\text {th }}$ day of storage lowest PLW was observed in $\mathrm{A}_{3} \mathrm{C}_{3}$ (2.99 $\%$ ) interaction and was at par with $\mathrm{A}_{1} \mathrm{C}_{3}$ (3.46\%), $\mathrm{A}_{2} \mathrm{C}_{3}$ (3.50\%), $\mathrm{A}_{3} \mathrm{C}_{2}$ (3.07\%), $\begin{array}{llllll}\mathrm{A}_{4} & \mathrm{C}_{1} & (3.05 \%), & \mathrm{A}_{4} & \mathrm{C}_{2} & (3.58 \%)\end{array}$ $\mathrm{A}_{4} \mathrm{C}_{3} \quad(3.31 \%)$ interactions. At $9^{\text {th }}$ day of storage lowest PLW was recorded in $\mathrm{A}_{3} \mathrm{C}_{2}$ (7.04 \%) interaction and was at par with $\mathrm{A}_{1} \mathrm{C}_{1}(7.72 \%)$ interaction.

\section{Moisture content}

Moisture loss may be due to respiration and transpiration of jackfruit bulbs during storage. Observation analogous to present findings have been reported by Phutankar (2013) and Khude (2012) in jackfruit.

\section{Spoilage (\%)}

Increase in spoilage during storage may be due to increase in microbial contamination which can also confirmed from the results presented in Tables 6 and 7, regarding bacterial and fungal counts. Spoilage increased with increase in storage period was observed by A. Abd-elghany (2012) in pomegranate fruits.

Singh et al., (2014) reported decrease in spoilage of guava fruits with increase in ascorbic acid concentration from $25 \mathrm{ppm}$ to 100 ppm. Microbial load decrease with increase in acidic condition, hence spoilage decreased with increase in ascorbic acid concentration.

In case of calcium chloride treatment, decrease in spoilage at $6^{\text {th }}$ day of storage may be due to their positive role in delaying the senescence of fruits by maintaining cell wall integrity and thus lowering the spoilage. Similar findings have been reported by Mahajan et al., (2011) when calcium concentration of 1,2 and 3 percent used in guava fruits. Rajkumar et al., (2005) when used calcium chloride of 1, 2, 3 and 4 percent in papaya fruits noticed that, spoilage decreased at 2 percent calcium chloride concentration and then increased at 3 percent of calcium chloride concentration.

In case of interaction significantly lowest spoilage was recorded in $\mathrm{A}_{4} \mathrm{C}_{2} \quad(5.37 \%)$ interaction at $6^{\text {th }}$ day of storage and was superior over other interactions.

\section{Shelf-life (days)}

Significantly maximum shelf life at $\mathrm{C}_{2}$ may be due to good cell wall integrity as a consequence of influx of calcium that could have helped in thickening of calcium pectate in the cell wall that assisted in prolonged shelf life.

Similar finding was noticed by Rajkumar et $a l .$, (2005) in papaya fruit.

In case of interaction, it is evident from the data that maximum shelf life (6 days) was recorded in bulbs treated with combination of 1.5 percent of ascorbic acid and 0.5 percent of calcium chloride concentration i.e., $\mathrm{A}_{4} \mathrm{C}_{2}$ interaction and significantly superior over rest of the interactions. This result is in agreement with Asrey (2006) in strawberry. 


\section{Bacterial count}

Increase in bacterial count may be due to increase in aerial microbes with increase in storage period. In case of ascorbic acid treatment, it is revealed from the data that, at 0,3 and $6^{\text {th }}$ day of storage non-significant results were found. Decrease in bacterial load with increase in ascorbic acid concentration may be due to increase in acidity and decrease in $\mathrm{pH}$ with increase in ascorbic acid concentration which results in unfavourable conditions for the growth of bacteria. Observations identical to present findings were also recorded by Boodia et al., (2009) and Acedo et al., (2013) in jackfruit.

In case of calcium chloride treatment, nonsignificant results were found at 0,3 and $6^{\text {th }}$ day of storage. At $9^{\text {th }}$ day of storage lowest bacterial count was observed in $\mathrm{C}_{2}$ $\left(10.81 \times 10^{-} \square\right)$ treatment and was significantly superior over other treatments.

In case of interaction of ascorbic acid and calcium chloride treatments it was that, minimum bacterial load was recorded in $\mathrm{A}_{4} \mathrm{C}_{2} \quad\left(0.50 \times 10^{-} \square\right)$ interaction, however it showed non-significant result and $\mathrm{A}_{2} \mathrm{C}_{3}$ $\left(7 \times 10^{-} \square\right)$ interaction recorded minimum load at $9^{\text {th }}$ day of storage and was significantly superior over other treatments.

\section{Fungal count}

Result revealed that, fungal count of fresh cut jackfruit bulbs increased throughout the storage period, irrespective of ascorbic acid and calcium chloride treatments.

Decrease in fungal count with increase in ascorbic acid may be the effect of increased concentration of ascorbic acid which affected the growth of fungus. Acedo et al., (2013) found reduced yeast and mould count in jackfruit by using ascorbic acid at 1.5 per cent.
In case of, effect of calcium chloride treatment and interaction of ascorbic acid and calcium chloride treatments non-significant results were found at $0,3,6$ and $9^{\text {th }}$ day of storage.

\section{Acknowledgements}

This work was supported by a grant from Dr. C.D. Pawar. I thank Dr. P. M. Haldankar and Dr. M. C. Kasture for guiding and supporting to complete research work.

\section{References}

A. Abd-elghany Nazmy, Samah I. Nasr and Hassan. M. Korkar 2012. Effects of polyolefin film wrapping and calcium chloride treatments on post harvest quality of "Wonderful" pomegranate fruits. Journal of Horticultural Science \& Ornamental Plants, 4(1): 07-17.

A.O.A.C. 1975. Official methods of analysis. Association of Official Analytical Chemists, Washington, D.C., $12^{\text {th }}$ Edn. pp. 15-18.

Acedo, J. Z., D. A. C. Varron, I. C. Emnace, R. D. Lauzon and Jr. A. L. Acedo 2013. Antimicrobial effects of ascorbic acid and calcium lactate in freshcut jackfruit (Artocarpus heterophyllus Lam.). Acta Hort., 989:199-208.

Asrey, R., R. Singh and S. Kumar 2006. Postharvest treatment and shelf life of Chandler strawberry. Ind. Fd. Packer, 22(2): 43-46.

Azad, A. K., 2000. Genetic diversity of jackfruit in Bangladesh and development of propagation methods. $\mathrm{Ph} . D$. Thesis (unpublished), submitted to University of Southampton, UK.

Bailey, L. H., 1942. The Standard Encyclopedia of Horticulture. The Macmillan Co. New York. 401-402.

Boodia Navindra, Arvind Ruggoo and Hassina Boodoo B. 2009. Effects of antibrowning agents on the shelf life of 
fresh-cut green jackfruit (Artocarpus heterophyllus Lam.). Journal of Applied Horticulture, 11(1): 35-40.

Gunasena, H. P. M., K. P. Ariyadasa, A. Wikramasinghe, H. M. W. Herath, P. Wikramasinghe and S. B. Rajakaruna 1996. Manual of Jack Cultivation in Sri Lanka. Forest Information Service, Forest Department: 48.

Khude, S., 2012. Effect of packaging on shelf-life of jackfruit (firm flesh) bulbs at different storage conditions. A M.Sc. (Hort.) thesis (unpublished), submitted to the Dr. B. S. Konkan Krishi Vidyapeeth, Dapoli, Dist. Ratnagiri, Maharashtra State.

Mahajan, B. V. C., B. S. Ghuman and Harsimrat K. Bons 2011. Effect of postharvest treatment of calcium chloride and gibbrellic acid on storage behaviour and quality of guava fruits. Journal of Horticulture Science \& Ornamental Plants, 3(1): 38-42.

Merrill, E. D., 1976. A Flora of Manila. Manila: Bu. of Printing. 369p.

Panase, V. G., and P. V. Sukhtame 1985. Statistical methods for agricultural workers, I. C. A. R. New Delhi.

Phutankar, N. R., 2013. Effect of packaging and storage conditions on storage of jackfruit (Artocarpus heterophyllus Lam.) firm flesh bulbs. A M.Sc. (Hort.) thesis (unpublished), submitted to the Dr. B. S. Konkan Krishi Vidyapeeth, Dapoli, Dist. Ratnagiri, Maharashtra State.

Rajkumar, M., Karuppaiah and R. Kandasamy 2005. Effect of calcium and gibberlic acid on post-harvest behavior of papaya Cv. Co2. Indian J. Hort., 62(4): 327331.

Sexena, A., Singh A. and P. S. Raju 2008. Use of modified atmosphere packaging to extend shelf-life of minimally processed jackfruit (Artocarpous heterophyllus L.) bulbs. Journal of Food Engineering, 87(4): 455-466.

Singh Gill, K., H. S. Dhaliwal and B. V. C. Mahajan 2014. Effect of post-harvest treatment of ascorbic acid on shelf life and quality of guava (Psidium guajava L.) cv. Allahaba safeda. Int. J. Agric. Sc \& Vet. Med. 2(1): 130-141.

\section{How to cite this article:}

Madhu M. Chulaki, C.D. Pawar and Salma Mustafa Khan. 2017. Influence of Ascorbic Acid and Calcium Chloride on Physical Parameters and Microbial Count of Firm Flesh Jackfruit Bulbs during Refrigerated Storage. Int.J.Curr.Microbiol.App.Sci. 6(10): 1101-1111. doi: https://doi.org/10.20546/ijcmas.2017.610.134 